\title{
The ACURATE neo2 valve system for transcatheter aortic valve implantation: 30-day and 1-year outcomes
}

\author{
Helge Möllmann ${ }^{1} \cdot$ David M. Holzhey $^{2} \cdot$ Michael Hilker $^{3} \cdot$ Stefan Toggweiler $^{4} \cdot$ Ulrich Schäfer $^{5} \cdot$ Hendrik Treede $^{6}$. \\ Michael Joner ${ }^{7,8} \cdot$ Lars Søndergaard $^{9} \cdot$ Thomas Christen $^{10} \cdot$ Dominic J. Allocco ${ }^{10}$ • Won-Keun Kim ${ }^{11}$
}

Received: 11 March 2021 / Accepted: 26 May 2021 / Published online: 20 June 2021

(c) The Author(s) 2021

\begin{abstract}
Background Transcatheter aortic valve implantation (TAVI) has become standard treatment for elderly patients with symptomatic severe aortic valve stenosis. The ACURATE neo AS study evaluates 30-day and 1-year clinical and hemodynamic outcomes in patients treated with the ACURATE neo2 valve.

Methods The primary endpoint of this single-arm multicenter study is 30-day all-cause mortality. Other key endpoints include device performance, echocardiographic measures assessed by an independent core laboratory, and VARC-2 clinical efficacy and safety endpoints through 12 months.

Results The study enrolled 120 patients (mean age $82.1 \pm 4.0$ years; $67.5 \%$ female, mean baseline STS score $4.8 \pm 3.8 \%$ ). The VARC- 2 composite safety endpoint at 30 days occurred in $13.3 \%$ of patients. All-cause mortality was $3.3 \%$ at 30 days and $11.9 \%$ at 1 year. The 30 -day stroke rate was $2.5 \%$ (disabling stroke 1.7\%); there were no new strokes between 30 days and 12 months. The rate of permanent pacemaker implantation was $15.0 \%$ (18/120) at 30 days and $17.8 \%(21 / 120)$ at 1 year. No patients required re-intervention for valve-related dysfunction and there were no cases of valve thrombosis or endocarditis. Patients demonstrated significant improvement in mean aortic valve gradient (baseline $38.9 \pm 13.1 \mathrm{mmHg}, 1$ year $7.8 \pm 3.5 \mathrm{mmHg} ; P<0.001$ in a paired analysis). In the overall population, paravalvular leak was evaluated at 1 year as none/ trace in $60.5 \%$, mild in $37.0 \%$, and moderate in $2.5 \%$; no patients had severe PVL.

Conclusions One-year outcomes from the ACURATE neo AS study support the safety and performance of TAVI with the ACURATE neo2 valve.
\end{abstract}

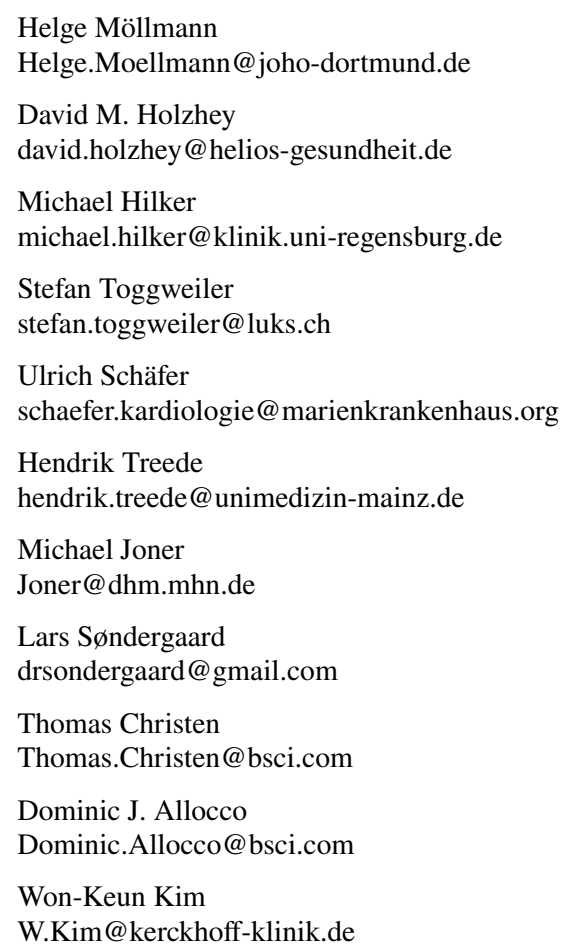

Department of Internal Medicine I, St.-Johannes-Hospital Dortmund, Johannesstraße 9-13, 44137 Dortmund, Germany

2 Department of Cardiovascular Surgery, Heart Center Leipzig, Leipzig, Germany

3 Klinik Für Herz-, Thorax und Herznahe Gefäßchirurgie, Universität Regensburg, Regensburg, Germany

4 Department of Cardiology, Luzerner Kantonsspital I LUKS, Luzern, Switzerland

5 Center for Internal Medicine, Marienkrankenhaus, Hamburg, Germany

6 Department of Cardiovascular Surgery, University Hospital Mainz, Mainz, Germany

7 Deutsches Herzzentrum München, Technische Universität München, Munich, Germany

8 DZHK (German Centre for Cardiovascular Research), Munich Heart Alliance, Munich, Germany

9 Department of Cardiology, Rigshospitalet, Copenhagen, Denmark

10 Boston Scientific, Marlborough, MA, USA

11 Department of Cardiology/Cardiac Surgery, Kerckhoff Heart Centre, Bad Nauheim, Germany 


\section{Graphic Abstract}

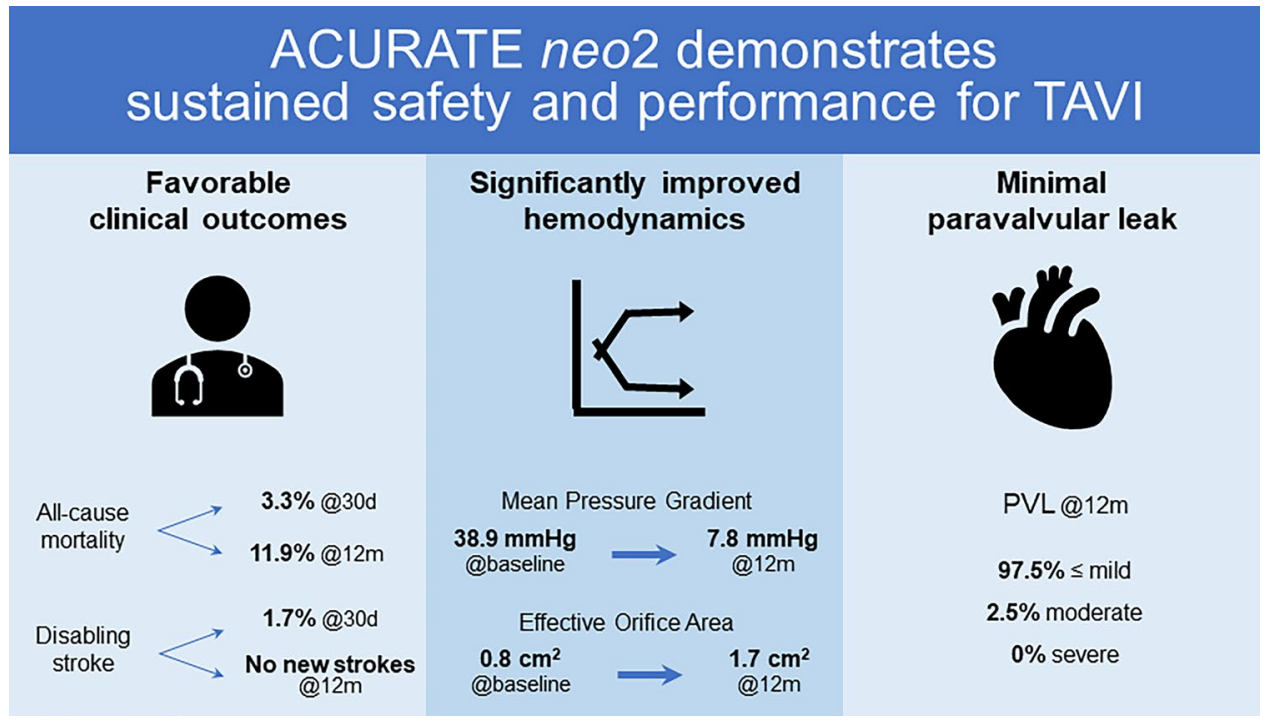

Keywords Aortic valve stenosis - Transcatheter aortic valve replacement - Transfemoral aortic valve implantation . Paravalvular regurgitation

\section{Introduction}

Transcatheter aortic valve implantation (TAVI), once reserved for patients who were inoperable or at high risk for surgical valve replacement, has recently been extended to intermediate- or low-risk populations. While clinical outcomes following TAVI are often comparable to those achieved surgically [1-4], there is some evidence that postTAVI complications such as patient-prosthesis-mismatch (PPM), paravalvular leak (PVL), and permanent pacemaker implantation (PPI) are associated with increased long-term mortality [5-8]. The self-expanding ACURAT E neo valve (Boston Scientific, Marlborough, MA) was designed to mitigate the risk of some of these complications: the supra-annular leaflet positioning contributes to lower gradients, the lower crown protrudes only minimally into the left ventricular outflow tract (LVOT) to minimize conduction system interference, and the integrated internal and external porcine pericardium sealing skirts reduce PVL [9]. ACURATE neo has demonstrated favorable clinical and echocardiographic outcomes, with low rates of mortality and PPI [10, 11]. However, two recent investigator-initiated studies which randomized patients to ACU RATE neo versus a later-generation competitor device (Sapien 3 and EvolutR/PRO) found a higher incidence of moderate or greater PVL in patients treated with ACURAT E neo, which contributed to its missing the non-inferiority primary endpoints $[12,13]$.
The ACURATE neo2 valve is an evolution of the ACU RATE neo valve (Fig. 1). The simplified implant procedure and supra-annular valve positioning are preserved, while the sealing skirts have been augmented to further reduce the PVL rate. Here we report the results of the ACURATE neo AS study, which focused on clinical and core laboratoryassessed echocardiographic outcomes after 30 days and 12 months in patients with severe symptomatic aortic stenosis treated with the next-generation ACURATE neo2 valve.

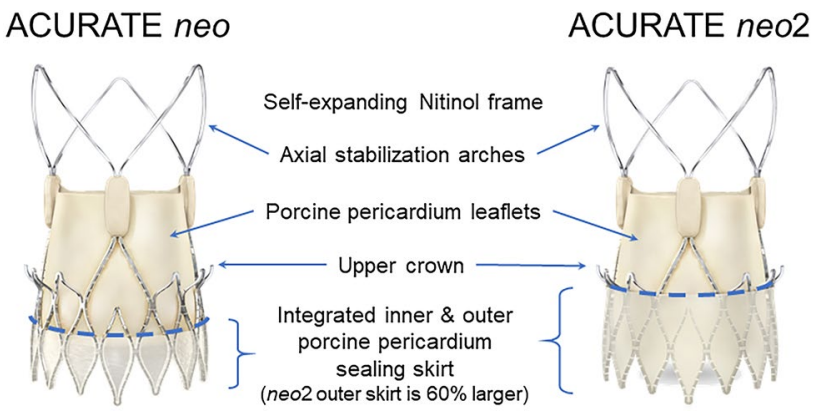

Fig. 1 The ACURATE valve family. ACURATE neo and ACURATE neo 2 are transcatheter self-expanding bioprosthetic aortic valves comprised of a nitinol frame with axial, self-aligning stabilization arches and supra-annular porcine pericardium leaflets. ACURATE neo2 represents an evolution of the valve design in that it features an enhanced sealing skirt to further reduce paravalvular leak 


\section{Methods}

\section{Study design and device details}

The ACURATE neo AS study was a single arm, prospective, non-randomized study conducted at European centers (see Supplementary Table S1). The study enrolled patients with severe symptomatic aortic stenosis for whom conventional aortic valve replacement was considered high risk for mortality or who were not operable as determined by a heart team consisting of a cardiologist and a surgeon. The study excluded patients with bicuspid aortic valves or previously implanted aortic bioprosthetic valves. Full inclusion and exclusion criteria are detailed in Supplementary Table S2. The protocol was approved by the locally appointed institutional review boards/ethics committees. The study was registered at ClinicalTrials.gov (NCT02909556) and was conducted in accordance with the International Conference for Harmonization Good Clinical Practice (ICH-GCP) regulations and guidelines and the ethical principles outlined in the Declaration of Helsinki. All patients gave written informed consent.

Like its predecessor, the CE-marked ACURATE neo aortic bioprosthesis, the ACURATE neo 2 valve is a transcatheter self-expanding bioprosthetic aortic valve comprised of a nitinol frame with axial, self-aligning stabilization arches and featuring supra-annular porcine pericardium leaflets (Fig. 1). The integrated internal and external porcine pericardium sealing skirts are designed to conform to irregular calcified anatomy with the goal of reducing PVL; the outer skirt on ACURATE neo 2 has been extended and is $60 \%$ larger than the skirt on ACURATE neo. The valve prosthesis is available in three different sizes ( $\mathrm{S}: 21 \mathrm{~mm} \leq$ annulus diameter $\leq 23 \mathrm{~mm}$, M: $23 \mathrm{~mm}<$ annulus diameter $\leq 25 \mathrm{~mm}$, and L: $25 \mathrm{~mm}<$ annulus diameter $\leq 27 \mathrm{~mm}$ ). In this study, the size $\mathrm{L}$ valve was only available after enrollment of the first 30 patients had been completed. Valve sizing was assessed by computerized tomography (CT); final size selection was at the operators' discretion.

\section{Clinical endpoints and outcomes analyses}

The primary endpoint of the study was the incidence of all-cause mortality in the intent-to-treat (ITT) population at 30 days. Key secondary endpoints included the rate of clinical events as defined per Valve Academic Research Consortium (VARC)-2 guidelines [14] at discharge/7 days, 30 days, and 12 months, and the VARC-2 safety composite at 30 days. Hemodynamic function, including effective orifice area, mean transprosthetic gradient, and aortic regurgitation, were assessed at discharge/7 days, 30 days, and 12 months of follow-up. Device and procedural success were also evaluated (see Supplementary Table S3 for definitions), in addition to functional improvement from baseline as per New York Heart Association (NYHA) Functional Classification.

To evaluate possible structural valve dysfunction, a post hoc evaluation of patients' longitudinal change in valve hemodynamics between 30 days and 1 year was performed (discharge/7-day data were used if 30-day data were not available). Criteria for hemodynamic valve dysfunction (HVD) were adapted from the recently published VARC-3 standardized definitions [15]. Morphological valve deterioration (Stage 1) is not reported here, as these data were not systematically collected in the ACURATE neo AS study. The definition of moderate HVD (Stage 2) is as follows, with changes for severe HVD (Stage 3 ) in brackets: increase in mean transvalvular gradient $\geq 10 \mathrm{mmHg}\{\geq 20 \mathrm{mmHg}\}$ resulting in mean gradient $\geq 20 \mathrm{mmHg}\{\geq 30 \mathrm{mmHg}\}$ with concomitant decrease in EOA $\geq 0.3 \mathrm{~cm}^{2}$ or $\geq 25 \%\{\geq 0.6$ $\mathrm{cm}^{2}$ or $\left.\geq 50 \%\right\}$ and/or decrease in DVI $\geq 0.1$ or $\geq 20 \%\{\geq 0.2$ $\mathrm{cm}^{2}$ or $\left.\geq 40 \%\right\}$, OR new occurrence or increase of $\geq 1$ grade $\{\geq 2$ grades $\}$ of transvalvular aortic regurgitation resulting in $\geq$ moderate \{severe $\}$ transvalvular aortic regurgitation.

An independent Data Monitoring Committee (DMC) was initially responsible for review of aggregate safety data up to 12 months; in April 2018 an independent Clinical Events Committee (CEC) assumed responsibility for adjudication of all reported VARC-2 endpoint events. All VARC-2 safety events were $100 \%$ monitored. To minimize bias and inconsistencies, all available echocardiographic data at baseline, discharge, and 30-day and 12-month follow-up were evaluated by an independent core laboratory (MedStar Health Research Institute, Hyattsville, MD).

\section{Statistical methods}

The study employed an optimal two-stage design, with sample size calculations based on an expected 30-day mortality rate of $10 \%$, based on literature review, and a one-sided alpha of 5\%. If three or more deaths occurred in the first stage of the study ( $n=30$ patients), the study could be terminated by the DMC. Per protocol, 30-day safety analyses were performed in the ITT population, which includes all enrolled patients in whom valve implantation was attempted. Clinical outcomes at 12 months were evaluated in those patients who received an ACURATE neo valve. Echocardiographic paired analyses were performed in the cohort of patients with core laboratory-adjudicated data available at baseline, 30 days, and 12 months post procedure.

Baseline and outcome variables were summarized using descriptive statistics where appropriate. For the comparison of categorical variables, statistical differences were assessed using a Chi-squared test or a Fisher's exact test, as appropriate. For the comparison of continuous variables, 
the Student's t test or analysis of variance was used. All statistical analyses were two-sided with an alpha level of 5\%. Statistical analyses were performed with SAS software (SAS Institute Inc., Cary, NC), version 9.3 or later.

\section{Results}

\section{Study population}

The study enrolled 120 patients between December 2016 and November 2017 at nine European centers. All patients were implanted with the ACURATE neo2 Aortic Valve System, so that the ITT and implanted populations were the same (in two patients an ACURATE neo 2 valve was initially implanted, but the patients subsequently underwent valvein-valve implantation with a non-study valve). Clinical follow-up data at 30 days were available for $98.3 \%$ of enrolled patients (118/120) and 12-month follow-up data were available for $92.5 \%$ (111/120). Two patients withdrew consent prior to 30-day follow-up, and an additional seven patients withdrew from the study between 30 days and 12 months.

Study patients were generally representative of patients treated in European contemporary practice (Table 1). The mean age of the study population was 82.1 years and the majority (67.5\%) were female. The mean EuroSCORE II was $4.7 \pm 3.8 \%$ and the mean STS score was $4.8 \pm 3.8 \% ; 11.7 \%$ of patients had an STS score $\geq 8 \%$. Eight patients $(6.7 \%)$ had a pacemaker at baseline, and a conduction abnormality was present at baseline in $47.5 \%$ of patients. Nearly all patients $(119 / 120 ; 99.2 \%)$ were classified as NYHA Functional Class III or IV at baseline, and $69.2 \%$ had a history of coronary artery disease. Mean AV gradient at baseline was $40.3 \pm 14.1 \mathrm{mmHg}$ and the mean aortic valve area (effective orifice area, EOA) was $0.74 \pm 0.2 \mathrm{~cm}^{2}$.

Per protocol, patients were to be prescribed dual antiplatelet therapy (DAPT) for 6 months post-TAVI, and aspirin for life; anticoagulation therapy was administered according to the usual practice at each site. At discharge, $56 \%$ of patients were on DAPT and $44 \%$ were taking anticoagulants. DAPT usage was $53 \%$ at 30 days and $28.0 \%$ at 6 months; anticoagulant usage was $36 \%$ at 30 days and $20 \%$ at 6 months.

\section{Clinical outcomes}

The median total procedure time was $48.5 \mathrm{~min}$. The most commonly implanted valve size was M ( $45 \%$ of cases). Balloon pre-dilatation was performed in $95.8 \%$ of patients; post-dilatation was performed in $32.5 \%$. The rate of procedural success was $97.5 \%$ (117/120). Although a single study valve was implanted in every patient, due to an inability to properly seat the valve in the annulus in two cases (one
Table 1 Patient demographics and baseline characteristics

\begin{tabular}{ll}
\hline Variable & $N=120$ \\
\hline Age, years & $82.1 \pm 4.0$ \\
Gender, female & $67.5(81)$ \\
Risk assessments & \\
STS Score, \% & $4.8 \pm 3.8$ \\
STS score $\geq 8 \%$ & $11.7(14)$ \\
EuroSCORE II & $4.7 \pm 3.8$ \\
NYHA Class III or IV & $99.2(119)$ \\
Medical history & \\
COPD, moderate or severe & $10(12)$ \\
Diabetes mellitus, medically treated & $27.5(33)$ \\
History of coronary artery disease & $69.2(83)$ \\
Porcelain aorta & $5.8(7)$ \\
History of cerebrovascular disease & $3.3(4)$ \\
Prior stroke / TIA & $10.8(13)$ \\
History of atrial fibrillation & $25.0 \%(30)$ \\
Previous cardiovascular interventions & \\
Prior PTCA & $4.2(5)$ \\
Prior PTCA with stenting & $26.7(32)$ \\
Prior CABG & $5.8(7)$ \\
Prior implanted pacemaker & $6.7(8)$ \\
Conduction abnormality at baseline & \\
Any conduction abnormality & $47.5 \%(57)$ \\
AV block, 1st degree & $15.0 \%(18)$ \\
LBBB & $10.8 \%(13)$ \\
RBBB & $8.3 \%(10)$ \\
Echocardiographi meeasurem & \\
&
\end{tabular}

Echocardiographic measurements (core laboratory adjudicated)

Aortic valve area (effective orifice area), $\mathrm{cm}^{2} \quad 0.74 \pm 0.2$

Mean aortic valve gradient, $\mathrm{mmHg} \quad 40.3 \pm 14.1$

Peak aortic valve gradient, $\mathrm{mmHg} \quad 65.9 \pm 21.4$

Left ventricular ejection fraction, $\% \quad 55.8 \pm 10.1$

Aortic regurgitation $\geq$ moderate $^{\mathrm{a}} \quad 6.1(7 / 115)$

Mitral regurgitation $\geq$ moderate $^{\mathrm{b}} \quad 8.3(9 / 108)$

Values are mean \pm standard deviation $(n)$ or $\%(n)$

$A V$ atrioventricular, $C A B G$ coronary artery bypass graft, $C O P D$ chronic obstructive pulmonary disease, $\angle B B B$ left bundle branch block, NYHA New York Heart Association, PTCA Percutaneous transluminal coronary angioplasty, $R B B B$ right bundle branch block, STS Society of Thoracic Surgeons, TIA transient ischemic attack

${ }^{a}$ Evaluated as 'moderate' in four patients (3.5\%) and 'moderately severe' in three patients $(2.6 \%)$

'Evaluated as 'moderate' in seven patients (6.5\%) and 'severe' in two patients (1.9\%)

valve embolization and one valve dislodgement/migration) a second non-study transcatheter valve was used (ie, valvein-valve implantation); data from these patients are included in the 30-day safety analysis, but not the 1-year analyses. In one patient, post-dilatation resulted in ventricular septal perforation and conversion to open heart surgery was necessary. There were no periprocedural deaths. Two patients 
(1.7\%) experienced disabling stroke prior to hospital discharge. There were no instances of coronary obstruction or cardiac tamponade in the periprocedural period, and no patients experienced a periprocedural ( $\leq 72 \mathrm{~h}$ post-procedure) myocardial infarction. Additional procedural details are presented in Table 2 .

The rate of all-cause mortality at 30 days in the ITT population (primary endpoint) was 3.3\% (4/120); none of the deaths were valve-related (Table 3 ). The 1-year all-cause mortality rate was $11.9 \%(14 / 118)$. The stroke rate was $2.5 \%$ at 30 days (3/120; periprocedural disabling stroke in two patients, and one additional non-disabling stroke on day 5). There were no additional stroke events between 30 days and 12 months. Major vascular complications occurred in four patients (3.3\%) through 30 days. The VARC-2 composite safety endpoint, which includes all-cause mortality, all stroke, major vascular complications, life-threatening or disabling bleeding, acute kidney injury (Stage 2/3), repeat procedure for valve-related dysfunction, and coronary obstruction requiring intervention at 30 days, occurred in $13.3 \%$ of patients. No patients in the study required reintervention for valve-related dysfunction, and there were no instances of prosthetic aortic valve thrombosis or endocarditis through 12-month follow-up.

At 30-day follow-up, left bundle branch block (LBBB) was reported in 24 patients $(23.5 \%)$. A total of $18 / 120$ patients $(15.0 \%)$ received a permanent pacemaker within 30 days (18/112 pacemaker-naïve patients; $16.1 \%)$. Among these patients, $8 / 18$ (44.4\%) had an underlying conduction disorder at baseline: four patients had right bundle branch block (two of these also presented with first degree AV block), three additional patients had first-degree AV block alone, and one patient had incomplete LBBB. Between 30 days and 12 months, three additional pacemakers were
Table 2 Procedural characteristics and periprocedural outcomes

\begin{tabular}{ll}
\hline Measure & $N=120$ \\
\hline Valve size implanted & $25.8(31)$ \\
$\mathrm{S}$ & $45.0(54)$ \\
$\mathrm{M}$ & $29.2(35)$ \\
$\mathrm{L}^{\mathrm{a}}$ & $48.5[22.5]$ \\
Total procedure time, minutes & $3.0[1.0]$ \\
Time from femoral insertion to withdrawal of delivery system, minutes & $95.8(115)$ \\
Balloon pre-dilatation performed & $23.0[4.0]$ \\
Maximum balloon diameter, mm & \\
Number of balloon inflations & $93.0(107)$ \\
1 & $5.2(6)$ \\
2 & $1.7(2)$ \\
3 & $32.5(39)$ \\
Post-dilatation performed & $23.0[2.0]$ \\
Maximum balloon diameter, mm & \\
Number of balloon inflations & $84.6(33)$ \\
1 & $15.4(6)$ \\
2 & $97.5(117)$ \\
Procedural success & $1.7(2)$ \\
Valve malpositioning (including valve migration, valve embolization, ectopic valve & \\
deployment) & \\
Ventricular septal perforation & \\
Coronary obstruction & $0.8(1)$ \\
Cardiac tamponade & $0.0(0)$ \\
MI $\leq 72$ h post-procedure & $0.0(0)$ \\
Life-threatening or disabling bleeding & $0.0(0)$ \\
Disabling stroke & $5.0(6)$ \\
\hline
\end{tabular}

Values are $\%(n / 120)$ or median $[\mathrm{IQR}]$

MI myocardial infarction

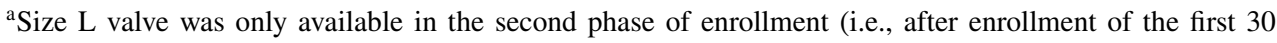
patients)

${ }^{b}$ Valve-in-valve implantation of a non-study valve required due to valve dislodgement/migration $(n=1)$, valve embolization $(n=1)$

${ }^{c}$ Perforation resultant from post-dilatation; patient was converted to open heart surgery 
Table 3 Safety outcomes

\begin{tabular}{lll}
\hline Clinical event & $\begin{array}{l}30 \text { Days } \\
N=120\end{array}$ & $\begin{array}{l}1 \text { Year } \\
N=118\end{array}$ \\
\hline VARC-2 early safety composite $^{\mathrm{a}}$ & $13.3(16)$ & - \\
All-cause mortality & $3.3(4)$ & $11.9(14)$ \\
Cardiovascular mortality & $3.3(4)$ & $9.3(11)$ \\
All stroke & $2.5(3)$ & $2.5(3)$ \\
Disabling stroke & $1.7(2)$ & $1.7(2)$ \\
Major vascular complications & $3.3(4)$ & $3.3(4)$ \\
Life-threatening/disabling bleeding & $5.0(6)$ & $8.5(10)$ \\
Acute kidney injury (stage 2 or 3) & $0.8(1)$ & $0.8(1)$ \\
Myocardial infarction > 72 h & $0.8(1)$ & $0.8(1)$ \\
Repeat procedure (surgery/interventional) for & $0.0(0)$ & $0.0(0)$ \\
$\quad$ valve-related dysfunction & & \\
Hospitalization for valve-related symptoms & - & $4.2(5)$ \\
$\quad$ or CHF & & \\
New permanent pacemaker & $15.0(18)$ & $17.8(21)$ \\
New-onset atrial fibrillation or atrial flutter & $5.8(7)$ & $8.5(10)$ \\
Coronary obstruction requiring intervention & $0.8(1)$ & $0.8(1)$ \\
Prosthetic aortic valve thrombosis & $0.0(0)$ & $0.0(0)$ \\
Prosthetic aortic valve endocarditis & $0.0(0)$ & $0.0(0)$ \\
\hline
\end{tabular}

Values are $\%(n)$; two patients required a valve-in-valve procedure with a non-study valve and thus are not included in the 1-year analyses

$B A V$ balloon aortic valvuloplasty; $C H F$ congestive heart failure

${ }^{a}$ Includes all-cause mortality, all stroke, major vascular complications, life-threatening or disabling bleeding, acute kidney injury (Stage 2/3), repeat procedure for valve-related dysfunction, and coronary obstruction requiring intervention

implanted, for a 1-year rate of $17.8 \%$ (18.8\% among pacemaker-naïve patients). A multivariate analysis did not identify any patient or procedural factors related to pacemaker implantation.

Functional improvement was evaluated per NYHA Functional Classification guidelines. At baseline, $95 \%$ of patients were classified as NYHA Functional Class III, and an additional $4.2 \%$ were NYHA Class IV (Fig. 2). Patients exhibited substantial improvement in function over the course of the study. From baseline to 12 months
post-TAVI, $91 \%$ of patients improved at least one functional class and $43 \%$ of patients improved at least two classes.

\section{Echocardiographic outcomes}

At 30 days, 115 patients were eligible for transthoracic (TTE) or transeosophageal echocardiography (TEE) assessment (data were unavailable for two patients due to withdrawal from study and three patients due to death prior to follow-up); 30-day echocardiographic data were evaluated for $104 / 115$ patients $(90.4 \%)$. At 12 months, 98 patients were eligible for echocardiographic followup (data unavailable for nine withdrawn patients and 13 deaths); 12-month echocardiographic assessment was completed for $89 / 98$ patients $(90.8 \%)$.

The overall as-treated population demonstrated excellent hemodynamics throughout the study (Supplementary Table S4). The mean AV gradient was $7.6 \pm 3.5 \mathrm{mmHg}$ and EOA was $1.7 \pm 0.4 \mathrm{~cm}^{2}$ at 1 year. At 12 -month followup, $60.5 \%$ of the overall study population had no/trace PVL, 37.0\% exhibited mild PVL, and 2.5\% had moderate PVL. No patients exhibited greater than moderate PVL at any time post procedure. A paired analysis was performed for patients with core laboratory-adjudicated echocardiographic data available at baseline, 30 days, and 12 months $(n=80)$. Patients demonstrated significant inter-individual improvement in mean $\mathrm{AV}$ gradient and EOA between baseline and 12 months $(P<0.001$ for both; Fig. 3a). At 30-day follow-up, PVL was evaluated as none/trace in $36.3 \%$ of patients in the paired cohort; this proportion improved to $60.0 \%$ at 12 months (Fig. 3b). We evaluated the change in valve hemodynamics between 30 days and 1 year to determine whether any patients had potential structural valve dysfunction. Based on this longitudinal assessment of hemodynamic function, no patients met the criteria for moderate or severe HVD (Supplementary Table S5).
Fig. 2 Change in New York Heart Association (NYHA) functional status. Patients exhibited marked improvement in NYHA class at 30 days postprocedure, which was maintained at 1 year

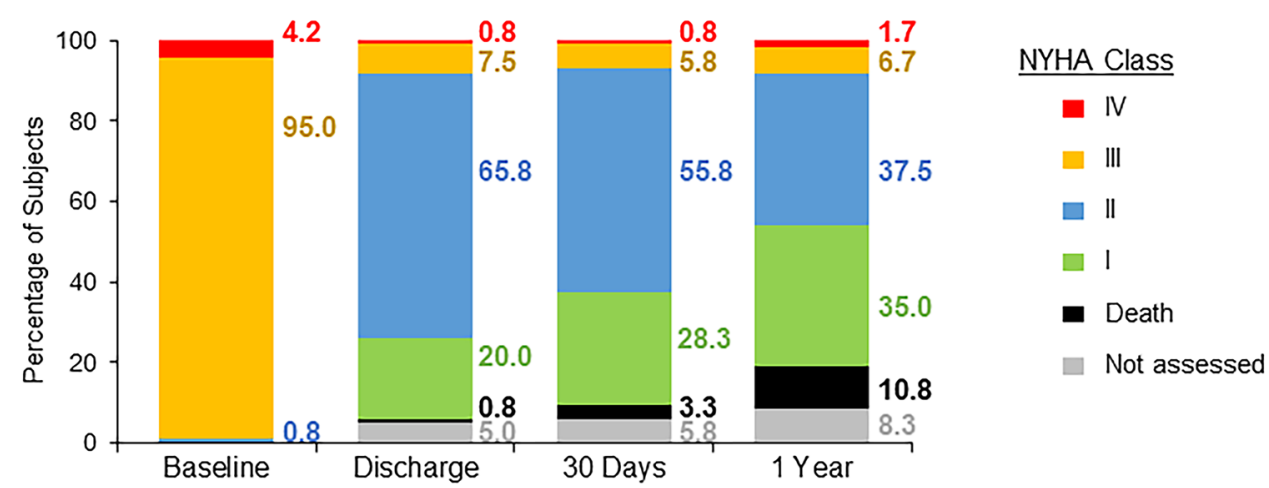


a) Valve hemodynamics

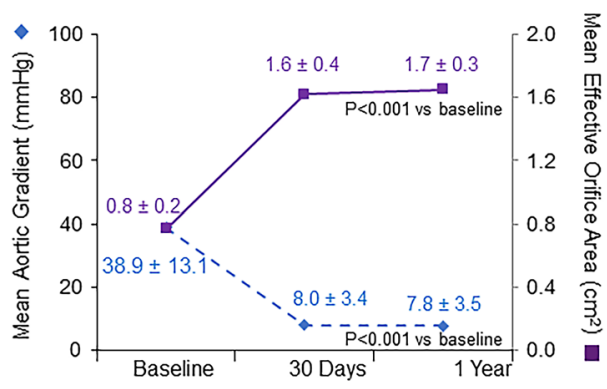

Fig. 3 Valve hemodynamics and paravalvular leak. Paired analyses were performed for patients with core laboratory-adjudicated echocardiographic data available at baseline, 30 days, and 12 months $(n=80)$. a Both mean aortic valve gradient and mean effective ori-

\section{Discussion}

The ACURATE neo 2 valve preserves many of the desirable attributes of the prior-generation ACURATE neo valve, and incorporates new features designed to mitigate some of the common complications associated with TAVI. A flexible delivery catheter allows for trackability through tortuous anatomy, radiopaque markers aid reference in positioning, and a simple two-step, top-down deployment method allows for stable and predictable release. Patients in the ACURAT E neo AS study achieved a high rate of procedural success $(97.5 \%)$. There were no reinterventions for valve-related dysfunction and a low rate of major vascular complications (3.3\%), comparable to the rates observed in recent studies with contemporary competitors such as Portico (5.5\%) [16], Evolut PRO (3.5\%) [17], and Sapien 3 (8.6\%) [18]. The ease of use of the ACURATE neo2 valve and the operators' prior experience with the ACURATE platform (over three-quarters of sites/investigators had treated patients with ACURAT E neo in the SAVI-TF study) may have contributed to the high rate of procedural success observed.

Overall, patients treated with ACURATE neo2 exhibited favorable early clinical outcomes. There was a low incidence of disabling stroke at 30 days (1.7\%), as in prior studies with ACURATE neo (1.2\% in SAVI-TF, $1.6 \%$ in the NEOPRO study) $[11,17]$. The VARC-2 composite safety endpoint rate at 30 days was similar or lower with ACURAT E neo2 (13.3\%) compared with prior studies of ACURAT E neo $(15.8 \%$ in the MORENA study [18]; $16.4 \%$ in the NEOPRO study [17]; $17.9 \%$ in Pellegrini, et al. [19]). Allcause mortality through 1 year was likewise comparable to recent studies with ACURATE neo and other contemporary valves $[10,19,20]$.

The ACURATE neo2 valve maintains a supra-annular leaflet position, allowing for a larger effective orifice area b) Paravalvular leak

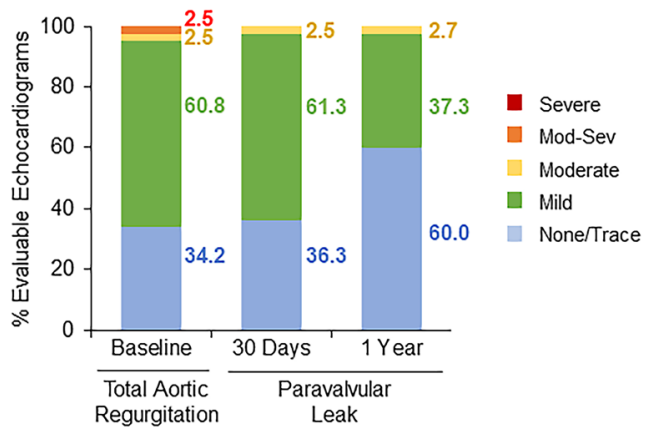

fice area (reported as time velocity integral [TVI] ratio) significantly improved from baseline to 1 year $(P<0.001$ for both). b Inter-individual improvement in paravalvular leak was observed over the course of the study

and lower gradients than valves with an intra-annular leaflet position. ACURATE neo AS patients demonstrated marked hemodynamic improvement at 30 days, with a significant change from baseline that was maintained through 12 months of follow-up (Fig. 3). While there have been no studies comparing ACURATE neo 2 to contemporary competitors, head-to-head comparisons of the prior-generation ACURATE neo to Sapien 3 in the MORENA study [18] and by Mauri et al. [20] have shown superior hemodynamics for ACURATE neo. Similarly, in the SCOPE I study more favorable gradients and valve areas were recorded for patients treated with supra-annular ACURATE neo compared to those treated with the intra-annular SAPIEN 3 device $\left(7 \mathrm{mmHg}\right.$ vs $11 \mathrm{mmHg}, P<0.0001 ; 1.73 \mathrm{~cm}^{2}$ vs $\left.1.46 \mathrm{~cm}^{2}, P<0.0001\right)$ [12].

In spite of its lower gradients and larger valve areas, ACU RATE neo missed the non-inferiority primary endpoint of early safety and clinical efficacy at 30 days in the SCOPE I study (absolute risk difference $7.1 \%$; upper $95 \%$ confidence limit $12.0 \% ; P=0.42$ ), due primarily to a higher incidence of patients with moderate or greater prosthetic valve regurgitation at 30 days (9\% vs 3\%; $P<0.001)$ [12]. In the SCOPE II study, wherein ACURATE neo was randomized against Evolut R/PRO, ACURATE neo missed the composite noninferiority primary endpoint for all-cause death and stroke at 1 year (absolute risk difference $1.8 \%$; upper one-sided $95 \%$ confidence limit $6.1 \% ; P=0.05$ ), due to a higher rate of cardiac mortality in the ITT population $(8.4 \%$ vs $3.9 \%$, $P=0.01$ ) [13]. ACURATE neo was associated with a higher 30 -day rate of moderate or greater PVL ( $10 \%$ vs $3 \%$; $P=0.002$ ), which may have contributed to the higher rate of cardiac death. As moderate or greater PVL has been linked to higher mortality rates [21-23], this is a continuing area of concern for TAVI.

The ACURATE neo 2 valve was designed to improve upon the existing pericardial sealing skirt. The extension 
of the outer skirt to the waist of the valve enhances the seal, further reducing PVL. Patients in the ACURATE neo AS study exhibited an overall rate of moderate PVL of $3.0 \%$ at 30 days, comparable to that observed with the competitor devices in SCOPE I (Sapien 3: 2.8\%) and SCOPE II (Evolut: $3.0 \%$ ). Patients with moderate PVL in the current study had severe calcification at baseline, highlighting the importance of exercising caution in patient selection. In addition to valve design, pre-procedural planning, including determination of optimal sizing and assessment of calcification, is crucial for a good outcome and may help to further lower the incidence of PVL [24]. Longer follow-up is warranted to determine if improvement in PVL translates into improved clinical outcomes.

A low rate of PPI has been a strength of the ACURATE neo valve platform. The valve is designed to extend cranially and does not protrude into the LVOT, reducing the risk of conduction system interference. As the overall device specifications and simplified implant procedure are preserved in the ACURATE neo2 valve design, it can also be expected to have a low PPI rate. The 30-day PPI rate in the current study $(15.0 \%)$ is higher than observed with ACURATE neo in SAVI-TF (8.3\%), or SCOPE I and II (10.0\% and $11.0 \%$, respectively). This finding may simply be due to chance, as the sample size analyzed in this manuscript is too small to provide a precise estimate of the PPI risk. Nonetheless, the PPI rate in the current study is within the range observed in recent trials with CoreValve/Evolut (US CoreValve High Risk: 19.8\%; Evolut Low Risk: 17.4\%; SCOPE II: 18.0\%) [1, 13, 25] and Sapien 3 (MORENA: 16.4\%; Mauri et al.: $15.2 \%)$ [18, 20]. However, such cross-study comparisons should be considered with caution, as the rate of PPI has been shown to vary widely across studies, and a number of factors may contribute to risk [26]. A multivariate analysis of typical risk factors for PPI (including baseline annular calcification, prior conduction disorders, and valve oversizing) did not reveal any strong association. As implanters become more familiar with ACURATE neo2 and take steps to optimize implantation technique, refining positioning using radiopaque markers, the PPI rate may decline.

The ACURATE neo AS study has a number of limitations. It was a single-arm, non-randomized study conducted in a relatively small population, and echocardiographic assessment was not available for all patients in the study at all time points, due primarily to differences in follow-up per local standard of care. Additionally, calcification data was qualitative only, and the protocol did not mandate core laboratory assessment of CT data, limiting the ability to assess the impact of annular calcification and valve sizing on clinical outcomes. Perhaps the greatest limitation is the absence of a direct comparator for ACURATE neo2. The currently enrolling ACURAT E IDE Study (NCT03735667) is a large prospective, multicenter, 1:1 randomized-controlled trial that will provide direct comparative data for ACURATE neo2 versus either a balloonexpandable (Sapien 3) or self-expanding (CoreValve / Evolut $\mathrm{R}$ / Evolut PRO) prosthetic valve.

\section{Conclusions}

Patients treated with the ACURATE neo 2 valve demonstrated good early clinical outcomes and showed significant improvement in valve hemodynamics at 30 days, which was maintained through 12-month follow-up. The overall rate of paravalvular leak was low, suggesting an improvement over prior studies with ACURATE neo.

Supplementary Information The online version contains supplementary material available at https://doi.org/10.1007/s00392-021-01882-3.

Acknowledgements The authors thank Songtao Jiang, MS (Boston Scientific Corporation) for statistical analysis; and MaryEllen Carlile Klusacek, $\mathrm{PhD}$ (Boston Scientific Corporation) for assistance in manuscript preparation.

Authors' contributions Boston Scientific was involved in conceiving and designing the research, in the collection, analysis, and interpretation of the data, and in the preparation and review of the manuscript draft.

All authors have participated in the work and have reviewed and agree with the content of the article.

Funding The ACURATE neo AS study was sponsored and funded by Symetis S.A., a subsidiary of Boston Scientific Corporation.

Data availability The data and study protocol for this clinical trial may be made available to other researchers in accordance with Boston Scientific's Data Sharing Policy on the Boston Scientific website.

\section{Declarations}

Conflict of interest H.M. reports receipt of honoraria or consultation fees from Abbott, Biotronik, Boston Scientific, Edwards Lifesciences, St. Jude Medical. D.H. is a proctor for Boston Scientific. M.H. reports receipt of honoraria or consultation fees from Boston Scientific and New Valve Technology. S.T. is a consultant and proctor for Boston Scientific, Abbott Vascular and New Valve Technology /Biosensors, has received speaker honoraria from Medtronic, institutional research grants from Boston Scientific and Fumedica, and is a board member of and holds equity in Hi-D Imaging. U.S. reports receipt of honoraria or consultation fees from Abbott, Biotronik, Boston Scientific, Edwards Lifesciences and Medtronic. H.T. reports receipt of honoraria or consultation fees from Medtronic, Biotronik, Boston Scientific, TriCares, JenaValve. M.J. did not report any conflicts of interest. L.S. has received consultant fees and institutional research grants from Boston Scientific. T.C. and D.A. are employees of and shareholders in Boston Scientific. W.K. reports receipt of honoraria or consultation fees from Abbott, Boston Scientific, Edwards Lifesciences, Medtronic.

Open Access This article is licensed under a Creative Commons Attribution 4.0 International License, which permits use, sharing, adaptation, distribution and reproduction in any medium or format, as long as you give appropriate credit to the original author(s) and the source, provide a link to the Creative Commons licence, and indicate if changes were made. The images or other third party material in this article are 
included in the article's Creative Commons licence, unless indicated otherwise in a credit line to the material. If material is not included in the article's Creative Commons licence and your intended use is not permitted by statutory regulation or exceeds the permitted use, you will need to obtain permission directly from the copyright holder. To view a copy of this licence, visit http://creativecommons.org/licenses/by/4.0/.

\section{References}

1. Adams DH, Popma JJ, Reardon MJ et al (2014) Transcatheter aortic-valve replacement with a self-expanding prosthesis. N Engl J Med 370:1790-1798. https://doi.org/10.1056/NEJMoa1400590

2. Smith CR, Leon MB, Mack MJ et al (2011) Transcatheter versus surgical aortic-valve replacement in high-risk patients. N Engl J Med 364:2187-2198. https://doi.org/10.1056/NEJMoa1103510

3. Mack MJ, Leon MB, Smith CR et al (2015) 5-year outcomes of transcatheter aortic valve replacement or surgical aortic valve replacement for high surgical risk patients with aortic stenosis (PARTNER 1): a randomised controlled trial. The Lancet 385:2477-2484. https://doi.org/10.1016/S0140-6736(15)60308-7

4. Leon MB, Smith CR, Mack MJ et al (2016) Transcatheter or surgical aortic-valve replacement in intermediate-risk patients. N Engl J Med 374:1609-1620. https://doi.org/10.1056/NEJMoa1514616

5. Head SJ, Mokhles MM, Osnabrugge RLJ et al (2012) The impact of prosthesis-patient mismatch on long-term survival after aortic valve replacement: a systematic review and meta-analysis of 34 observational studies comprising 27186 patients with 133141 patient-years. Eur Heart J 33:1518-1529. https://doi.org/10.1093/ eurheartj/ehs003

6. Pibarot P, Weissman N, Stewart W et al (2014) Incidence and sequelae of prosthesis-patient mismatch in transcatheter versus surgical valve replacement in high-risk patients with severe aortic stenosis: a PARTNER Trial Cohort A analysis. J Am Coll Cardiol 64:1323-1334. https://doi.org/10.1016/j.jacc.2014.06.1195

7. Jørgensen TH, Backer OD, Gerds TA et al (2019) Mortality and heart failure hospitalization in patients with conduction abnormalities after transcatheter aortic valve replacement. JACC Cardiovasc Interv 12:52-61. https://doi.org/10.1016/j.jcin.2018.10. 053

8. Faroux L, Chen S, Muntané-Carol G, et al (2020) Clinical impact of conduction disturbances in transcatheter aortic valve replacement recipients: a systematic review and meta-analysis. Eur Heart J 41:2771-2781. https://doi.org/10.1093/eurheartj/ehz924

9. Möllmann H, Diemert P, Grube E et al (2013) Symetis ACURATE $\mathrm{TF}^{\mathrm{TM}}$ aortic bioprosthesis. EuroIntervention 9:S107-S110. https:// doi.org/10.4244/eijv9ssa22

10. Kim W-K, Hengstenberg C, Hilker M et al (2018) The SAVITF registry: 1-year outcomes of the European post-market registry using the ACURATE neo transcatheter heart valve under real-world conditions in 1,000 patients. JACC Cardiovasc Interv 11:1368-1374. https://doi.org/10.1016/j.jcin.2018.03.023

11. Möllmann H, Hengstenberg C, Hilker M et al (2018) Realworld experience using the ACURATE neo ${ }^{\mathrm{TM}}$ prosthesis: 30 -day outcomes of 1000 patients enrolled in the SAVI-TF registry. EuroIntervention 13:e1764-e1770. https://doi.org/10.4244/ EIJ-D-17-00628

12. Lanz J, Kim W-K, Walther T et al (2019) Safety and efficacy of a self-expanding versus a balloon-expandable bioprosthesis for transcatheter aortic valve replacement in patients with symptomatic severe aortic stenosis: a randomised non-inferiority trial. The Lancet 394:1619-1628. https://doi.org/10.1016/S0140-6736(19) 32220-2

13. Tamburino C, Bleiziffer S, Thiele H et al (2020) Comparison of self-expanding bioprostheses for transcatheter aortic valve replacement in patients with symptomatic severe aortic stenosis: the SCOPE 2 randomized clinical trial. Circulation. https://doi. org/10.1161/CIRCULATIONAHA.120.051547

14. Kappetein AP, Head SJ, Genereux P et al (2012) Updated standardized endpoint definitions for transcatheter aortic valve implantation: the valve academic research consortium- 2 consensus document. J Am Coll Cardiol 60:1438-1454. https://doi.org/10.1016/j. jacc.2012.09.001

15. Genereux P (2021) Valve academic research consortium 3: updated endpoint definitions for aortic valve clinical research. J Am Coll Cardiol 77:2717-2746. https://doi.org/10.1016/j.jacc. 2021.02.038

16. Søndergaard L, Rodés-Cabau J, Hans-Peter Linke A et al (2018) Transcatheter aortic valve replacement with a repositionable selfexpanding prosthesis: the PORTICO-I trial 1-year outcomes. J Am Coll Cardiol 72:2859-2867. https://doi.org/10.1016/j.jacc.2018. 09.014

17. Pagnesi M, Kim W-K, Conradi L et al (2019) Transcatheter aortic valve replacement with next-generation self-expanding devices: a multicenter, retrospective, propensity-matched comparison of Evolut PRO versus accurate neo transcatheter heart valves. JACC Cardiovasc Interv 12:433-443. https://doi.org/10.1016/j.jcin. 2018.11.036

18. Husser O, Kim W-K, Pellegrini C et al (2017) Multicenter comparison of novel self-expanding versus balloon-expandable transcatheter heart valves. JACC Cardiovasc Interv 10:2078-2087. https://doi.org/10.1016/j.jcin.2017.06.026

19. Pellegrini C, Rheude T, Trenkwalder T et al (2019) One-year clinical outcome with a novel self-expanding transcatheter heart valve. Catheter Cardiovasc Interv 94:783-792. https://doi.org/10.1002/ ccd. 28144

20. Mauri V, Kim WK, Abumayyaleh M et al (2017) Short-term outcome and hemodynamic performance of next-generation selfexpanding versus balloon-expandable transcatheter aortic valves in patients with small aortic annulus: a multicenter propensitymatched comparison. Circ Cardiovasc Interv 10:e005013. https:// doi.org/10.1161/CIRCINTERVENTIONS.117.005013

21. Tamburino C, Capodanno D, Ramondo A et al (2011) Incidence and predictors of early and late mortality after transcatheter aortic valve implantation in 663 patients with severe aortic stenosis. Circulation 123:299-308. https://doi.org/10.1161/circulationaha. 110.946533

22. Van Belle E, Juthier F, Susen S et al (2014) Postprocedural aortic regurgitation in balloon-expandable and self-expandable transcatheter aortic valve replacement procedures: analysis of predictors and impact on long-term mortality: insights from the FRANCE2 Registry. Circulation 129:1415-1427. https://doi.org/ 10.1161/circulationaha.113.002677

23. Abdel-Wahab M, Zahn R, Horack M et al (2011) Aortic regurgitation after transcatheter aortic valve implantation: incidence and early outcome. Results from the German Transcatheter Aortic Valve Interventions registry. Heart 97:899-906. https://doi.org/10. 1136/hrt.2010.217158

24. Kim W-K, Möllmann H, Liebetrau C et al (2020) Effectiveness and safety of the ACURATE neo prosthesis in 1,000 patients with aortic stenosis. Am J Cardiol. https://doi.org/10.1016/j.amjcard. 2020.05.044

25. Popma JJ, Deeb GM, Yakubov SJ et al (2019) Transcatheter aortic-valve replacement with a self-expanding valve in low-risk patients. N Engl J Med 380:1706-1715. https://doi.org/10.1056/ NEJMoa1816885

26. Erkapic D, De Rosa S, Kelava A et al (2012) Risk for permanent pacemaker after transcatheter aortic valve implantation: a comprehensive analysis of the literature. J Cardiovasc Electrophysiol 23:391-397. https://doi.org/10.1111/j.1540-8167.2011.02211.x 